\title{
ESTUDO DE LIBERAÇÃO E PERMEAÇÃO IN VITRO DO DICLOFENACO DE DIETILAMÔNIO EM MICROEMULSÃO GEL-LIKE
}

\author{
José Alexsandro da Silva* \\ Departamento de Farmácia, Universidade Estadual da Paraíba, Rua Juvêncio Arruda, s/n, 58109-790 Campina Grande - PB, Brasil \\ Davi Pereira de Santana, Danilo Galindo César Bedor e Valeria Ferreira da Costa Borba \\ Departamento de Ciências Farmacêuticas, Universidade Federal de Pernambuco, Rua Prof. Arthur de Sá, s/n, 50739-520 Recife \\ - PE, Brasil \\ Ana Amélia Moreira Lira \\ Departamento de Fisiologia, Curso de Farmácia, Universidade Federal de Sergipe, São Cristóvão - SE, Brasil \\ Eryvaldo Socrates Tabosa do Egito \\ Departamento de Farmácia, Universidade Federal do Rio Grande do Norte, Natal - RN, Brasil
}

Recebido em 25/3/08; aceito em 25/2/09; publicado na web em 3/7/09

\begin{abstract}
IN VITRO RELEASE AND PERMEATION OF A DICLOFENAC DIETHYLAMINE FROM MICROEMULSION GEL-LIKE. The goal of this study was to produce and characterize a new microemulsion gel-like carrier system (MEG) by using the pseudoternary phase-diagram concept. The diclofenac diethylamine (DDA) was incorporated in the MEG and its in vitro release and permeation profiles were performed using Franz-type diffusion cells. The results revealed that the commercial DDA emulgel provided significantly higher $K_{p}$ of DDA (2.2-fold) as compared to the MEG. Similar data were obtained in the permeation studies in which DDA $K_{p} 4.7$-fold higher. Therefore, MEG presents higher potential as a topical delivery system for DDA when compared to the commercial DDA emulgel.
\end{abstract}

Keywords: diclofenac diethylamine; in vitro release; microemulsion gel-like.

\section{INTRODUÇÃO}

A administração tópica é conhecida como via alternativa da administração oral de ativos medicamentosos e oferece muitas vantagens como a ausência de efeito de primeira passagem, o fato de ser indolor e a facilidade de aplicação. ${ }^{1}$ Todavia, uma eficácia tópica só é obtida a partir de fármacos potentes com adequado grau de penetração na pele. ${ }^{2}$ Isto tem levado a um crescente interesse pela utilização de promotores de permeação em formulações tópicas, assim como o uso de sistemas de liberação de fármacos com esta capacidade promotora de permeação. ${ }^{3}$

As microemulsões (ME) são dispersões isotrópicas, transparentes, termodinamicamente estáveis, usualmente formadas por misturas de quatro componentes, água, óleo, tensoativo e cotensoativo. Apresentam grande potencial como sistemas de liberação e de direcionamento de fármacos, pelas propriedades de solubilizar fármacos hidrofílicos em meio lipofílico, lipofílicos em meio aquoso e anfifílicos na interface óleo/água. ${ }^{4-10}$ Além disso, estes sistemas apresentam capacidade de se formarem espontaneamente, podendo existir sob várias formas estruturais (globular a/o e o/a, bicontínuas, cúbicas ou lamelares), ${ }^{11,12}$ e atuam como promotores de permeação de vários fármacos, devido ao reduzido tamanho das gotículas formadas, assim como ao seu alto conteúdo de tensoativo, que desorganiza os lipídeos da pele. ${ }^{5}$

O diclofenaco de dietilamônio (DDA) é um anti-inflamatório não esteroidal (AINEs) capaz de formar micelas e cristais líquidos liotrópicos em água. ${ }^{13}$ Apresenta a capacidade de interagir com os fosfolipídeos da pele, contribuindo para aumentar a fluidez do mesmo no estrato córneo, fazendo com que os lipídios passem de uma forma cristalina ordenada para uma forma líquida desordenada, aumentando, portanto, a permeabilidade cutânea. ${ }^{5,7}$ Além disso, o

\footnotetext{
*e-mail: alexuepb@yahoo.com.br
}

DDA apresenta metabolismo hepático, tempo de meia-vida curto, ${ }^{14}$ não sofre metabolismo na pele, não induz tolerância e tem bom coeficiente de partição. ${ }^{15}$

Com base nas colocações acima e na importância do tema, o objetivo do presente trabalho foi a obtenção e a caracterização de uma ME, na forma gel (MEG) contendo DDA, como sistema promotor de permeação do fármaco, e a avaliação do seu perfil de liberação e permeação in vitro em comparação a uma formulação convencional emulgel comercial genérico.

\section{PARTE EXPERIMENTAL}

\section{Materiais}

Diclofenaco de dietilamônio (Nortec Química, Brasil), metanol e acetonitrila grau cromatográfico (J. T Baker), fosfato de potássio monobásico (Merck), água purificada do sistema Millipore Milli- ${ }^{\circledR}$ (Millipore Corporation - USA), membranas hidrofílicas de 0,45 $\mu \mathrm{m}$ de acetato de celulose, emulgel comercial de diclofenaco dietilamônio genérico $(11,6 \mathrm{mg} / \mathrm{g})$, miristato de isopropila (Henrifarma), polietilenoglicol-8 cáprico caprílico glicerídeo (Labrasol ${ }^{\circledR}$-Gattefossé), poligliceril 6-dioleato (Plurol Oleico ${ }^{\circledR}$-Gattefossé).

\section{Métodos}

Construção do diagrama de fases

O diagrama de fase foi obtido por inspeção visual das misturas dos ingredientes polietilenoglicol-8 cáprico caprílico glicerídeo (tensoativo) com poligliceril 6-dioleato (co-tensoativo) na proporção 2:1 e, a esta mistura, foi adicionado miristato de isopropila (fase oleosa) nas proporções de 1:9, 2:8, 3:7, 4:6, 5:5, 6:4, 7:3, 8:2 e 9:1. As titulações foram feitas com água purificada tipo Milli-Q ${ }^{\circledR}$, utilizando uma 
pipeta automática ajustada a $1000 \mu \mathrm{L}$, adicionando gota a gota, em temperatura ambiente. Durante a titulação a mistura foi homogeneizada por agitador magnético, por $3 \mathrm{~min}$, e as mudanças do aspecto visual contra um fundo preto foram observadas. Considerando-se as proporções dos componentes após as titulações aquosas plotaram-se os pontos em que se deu a formação de sistema isotrópico e opticamente transparente MEG. ${ }^{4,6,16-18} \mathrm{O}$ diagrama foi feito em duplicata.

\section{Seleção da formulação MEG}

A MEG foi selecionada, a partir de uma determinada área do diagrama de fases, na qual foi preparada a partir da mistura dos ingredientes polietilenoglicol-8 cáprico caprílico glicerídeo como tensoativo $(35,3 \%)$ com poligliceril 6-dioleato como cotensoativo $(17,6 \%)$ $\mathrm{e}$, a esta mistura, foi adicionado o miristato de isopropila como fase oleosa $(5,9 \%)$ e em seguida água ultrapura tipo Milli-Q ${ }^{\circledR}(41,2 \%)$. $\mathrm{O}$ diclofenaco de dietilamônio foi incorporado a este sistema, por simples solubilização, na concentração de $1,16 \%$. A formulação foi preparada $48 \mathrm{~h}$ antes dos testes de caracterização, para estabilização termodinâmica do sistema em temperatura ambiente. ${ }^{19}$

\section{Caracterização físico-química da MEG selecionada}

Análise do tamanho das gotículas (espalhamento de luz dinâmico)

A análise do tamanho das gotículas sub-microscópicas foi determinada através da técnica de espalhamento de luz dinâmico, também denominada espectroscopia de correlação de fótons (PCS), cuja técnica fornece o raio hidrodinâmico das partículas coloidais, o qual é calculado pela equação de Stokes-Einstein. ${ }^{17} \mathrm{~A}$ MEG foi analisada em um analisador de gotículas. As amostras foram colocadas na câmara de análise de modo que o feixe de laser atravessasse a dispersão em toda a sua extensão. A temperatura do sistema foi mantida a $20^{\circ} \mathrm{C}$, o comprimento de onda do laser foi de $532 \mathrm{~nm}$ e o índice de refração foi de acordo com o índice calculado de cada amostra analisada. Foram realizadas 10 determinações do diâmetro e índice de polidispersidade (IPD) das gotículas de cada amostra ( $n=3$ ), com a duração total de 5 min.

\section{Determinação da eficiência de encapsulação}

Para o doseamento do DDA na MEG, por espectrofotometria, foi preparada uma solução de $\approx 20 \mu \mathrm{g} \mathrm{mL}^{-1}$ de DDA ( $50 \mu \mathrm{L}$ de MEGDDA diluídos em 24,95 mL de metanol). As concentrações de DDA na MEG foram medidas a partir dos valores de absorbância, tendo como valor exato o valor correspondente ao padrão diário contendo $100 \mu \mathrm{g} \mathrm{mL} \mathrm{m}^{-1}$ (10 mg de DDA em $100 \mathrm{~mL}$ de metanol). A partir desta solução, foi realizada uma diluição com metanol de forma a obter uma solução de $20 \mu \mathrm{g} \mathrm{mL}^{-1}$. As leituras foram feitas em $282 \mathrm{~nm}$ segundo protocolo adotado no desenvolvimento e validação de metodologia analítica por espectrofotometria, ${ }^{2,20}$ cuja linearidade foi de 5 a $35 \mu \mathrm{g}$ $\mathrm{mL}^{-1}(\mathrm{r}=0,995)$, com uma precisão intra e inter corrida abaixo de $5 \%$ e uma exatidão próxima de $100 \%$, em todos os níveis de concentração (baixa, média e alta). Além disso, o método se mostrou seletivo, já que na varredura espectral (250-350 nm) a formulação MEG sem o DDA não apresentou absorbância em $282 \mathrm{~nm} .{ }^{20}$

A eficiência de encapsulação do DDA na MEG foi determinada utilizando-se $1 \mathrm{~mL}$ da formulação, a qual foi submetida à centrifugação $(11000 \mathrm{~g} \approx 14000 \mathrm{rpm})$ por $30 \mathrm{~min}$. Ao término do ciclo de centrifugação, foi feito o doseamento do sobrenadante, cuja percentagem de encapsulação foi calculada pela razão entre o teor do sobrenadante versus o teor na MEG x 100. ${ }^{2,20}$

\section{Condutividade ( $\underline{\sigma})$}

A $\sigma$ foi determinada em condutivímetro modelo Cambo HI 98129 da marca Hanna. O equipamento foi calibrado com uma solução de calibração de condutância específica de $1413 \mu \mathrm{S} \mathrm{cm}$ ç $^{-1}$ à temperatura de $25^{\circ} \mathrm{C} .{ }^{16}$

\section{Determinação do $\mathrm{pH}$}

$\mathrm{O} \mathrm{pH}$ da MEG foi avaliado utilizando-se um potenciômetro digital com eletrodo de vidro e sensor de temperatura, modelo Cambo HI 98129, da marca Hanna, previamente calibrado com soluções tampão $\mathrm{pH} 4,0$ e 7,0 a uma temperatura de $25 \pm 0,5^{\circ} \mathrm{C}$. O eletrodo de vidro foi introduzido diretamente no interior da formulação $(\approx 15 \mathrm{~mL})$ acondicionada em frasco de cintilação.

\section{Índice de Refração (IR)}

O IR foi determinado utilizando-se Refratômetro de Abbé, Carl Zeiss-Jena, aferido com água ultrapura Milli-Q ${ }^{\circledR}$ (IR 1,3325), à temperatura de $25 \pm 0,5^{\circ} \mathrm{C}$, e em triplicata. ${ }^{17}$

\section{Determinação da densidade relativa $(D R)$}

A DR foi determinada pelo método do picnômetro, seguindo-se a metodologia descrita na Farmacópeia Brasileira, ${ }^{21}$ utilizando-se como referência água ultrapura Milli- $\mathrm{Q}^{\circledR}$. Os picnômetros utilizados foram de $5 \mathrm{~mL}$ de capacidade e para retirar as bolhas de ar formadas no sistema foi utilizado o banho ultrassom (UltraSonic - USC 1400). A densidade foi obtida em ambiente com temperatura de 20,0 \pm $0,5^{\circ} \mathrm{C}$ e em triplicata.

\section{Determinação do potencial zeta}

A medida do potencial zeta $(\zeta \mathrm{mV})$ foi determinada com auxílio de um zetâmetro [Particle Sizing System by Light Scattering - Brookhaven Instruments Corporation, model EMI 9863 (Holtsville, NY, USA)], submetendo-se as gotículas da formulação a uma voltagem fixa e utilizando os valores fornecidos pelo aparelho para calcular o $\zeta \mathrm{mV}$. Para cada amostra $(\mathrm{n}=3)$ foram realizadas 15 medidas de mobilidade eletroforética. ${ }^{22}$

\section{Estudo de liberação e permeação in vitro do DDA na MEG}

O estudo de liberação e permeação in vitro do DDA na MEG foi conduzido utilizando-se células de difusão tipo Franz com área difusional de $1,15 \mathrm{~cm}^{2}$, volume de $\pm 6 \mathrm{~mL}$, usando membranas artificiais hidrofílicas de acetato de celulose $0,45 \mu \mathrm{m}$ para o estudo de liberação e pele da orelha de porco para o estudo de permeação. $\mathrm{O}$ compartimento receptor foi preenchido com tampão fosfato $\mathrm{pH} 7,4$ num sistema composto de seis células individuais conectado a um banho termostatizado à $37 \pm 0,5^{\circ} \mathrm{C}$ sob agitação constante de $100 \mathrm{rpm}$ em agitador magnético por um período de $12 \mathrm{~h}$ para perfil cinético de liberação e de $24 \mathrm{~h}$ para perfil cinético de permeação. As membranas foram colocadas na parte superior da célula receptora. No compartimento doador, foram aplicados $300 \mu \mathrm{L}$ da MEG (o que corresponde a $3480 \mu \mathrm{g}$ de DDA) diretamente sobre a membrana e, posteriormente, o sistema foi fechado. As amostras da solução receptora foram coletadas nos seguintes tempos para o perfil cinético de liberação: 0,$5 ; 1,0 ; 1,5$; 2,$0 ; 2,5 ; 3,0 ; 4,0 ; 6,0 ; 8,0 ; 10$ e 12 h e de 1,$0 ; 2,0 ; 3,0 ; 4,0 ; 6,0 ; 8,0$; $10 ; 12$ e 24 h para o perfil cinético de permeação. O volume total da fase receptora foi substituído a cada amostragem com tampão fosfato pH 7,4 para manutenção das condições sink do sistema. ${ }^{23}$

A pele da orelha de porco foi obtida e tratada de animal recentemente sacrificado em matadouro da região metropolitana do Recife, cuja pele foi retirada da cartilagem com um auxílio de pinça e bisturi, seguindo-se de remoção do tecido gorduroso remanescente, sendo em seguida seccionadas e congeladas. ${ }^{2}$

\section{Quantificação do DDA por CLAE-UV}

A quantidade de fármaco liberado no compartimento receptor foi quantificada por um sistema de cromatografia líquida de alta eficiência Shimadzu (Kyoto, Japão) configurado em $280 \mathrm{~nm}$. A separação cromatográfica foi conseguida usando-se uma coluna analítica tipo C18 150 
x 4,6 mm, 5 mm Shimpack ${ }^{\circledR}$ (Shimadzu, Kyoto, Japão) acoplada a uma pré-coluna C18 4,0 x 3,0 mm (Phenomenex, Torrance, CA, EUA). A fase móvel utilizada foi uma mistura de acetonitrila e tampão fosfato de sódio $20 \mathrm{mM}(70: 30$, v/v) ajustado a pH 3,0 com ácido fosfórico, na qual foi filtrado e degaseificado e bombeado em um fluxo de 1,2 $\mathrm{mL} \mathrm{min}^{-1}$. O forno da coluna foi ajustado para $40{ }^{\circ} \mathrm{C}$ e o volume de injeção foi $30 \mu \mathrm{L}$, com tempo de corrida total de $3,5 \mathrm{~min}$. O método de quantificação do DDA foi validado, com uma linearidade entre 0,05 e $20,0 \mu \mathrm{g} \mathrm{mL}^{-1}$ e coeficiente de correlação de 0,998 , limite de detecção de $0,003 \mu \mathrm{g} \mathrm{mL}^{-1}$ e limite de quantificação de $0,05 \mu \mathrm{g} \mathrm{mL} \mathrm{m}^{-1}$, com uma precisão intra e inter corrida abaixo de $5 \%$ e uma exatidão nos três níveis (baixa, média e alta concentração) próximo de $100 \%$.

A liberação e a permeação da MEG foi comparada com 300 mg do medicamento comercial genérico emulgel de diclofenaco de dietilamônio na mesma concentração.

\section{Cálculo do fluxo $(J)$ e do coeficiente de permeabilidade $\left(K_{p}\right)$ do DDA na MEG}

O $J$ foi calculado no steady-state a partir da porção linear da quantidade liberada por área versus tempo conforme dados da literatura. ${ }^{1,24,25}$

Já o $K_{p}$ foi calculado a partir do $J$ no steady-state versus a concentração de DDA no compartimento doador $\left(\mathrm{C}_{\text {doador }}\right)$ conforme Equação 1 sugerida por Ozguney et al.. ${ }^{1}$

$K_{p}=J / \mathrm{C}_{\text {doador }}$

\section{RESULTADOS E DISCUSSÃO}

\section{Diagrama de fases}

Durante o processo de produção do sistema MEG, selecionado a partir do diagrama de fases deste estudo, foi possível observar que a energia empregada e as proporções de seus componentes (óleo, água, tensoativo e cotensoativo) foram apropriadas para reduzir a tensão interfacial até certo ponto e foi capaz de conduzir a formação de um sistema homogêneo, límpido, translúcido e isotrópico característico de MEG. Dessa forma, foi possível definir com clareza a região de MEG como representada na Figura 1.

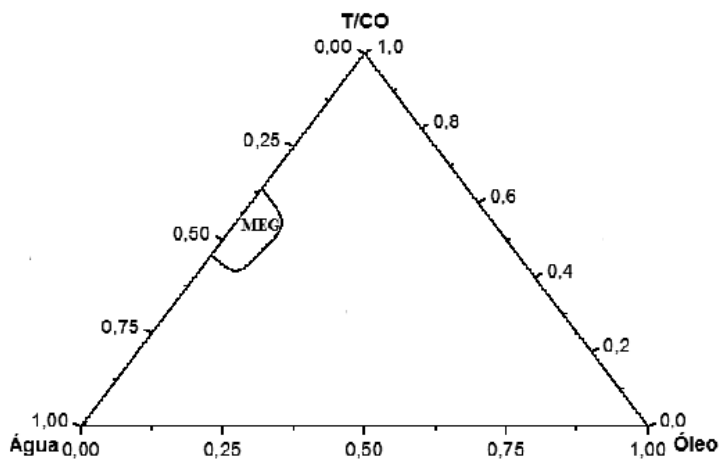

Figura 1. Diagrama de fases pseudo-ternário para o sistema polietilenoglico-8 cáprico caprílico gricerídeo $\left(\right.$ Labrasol $\left.^{\circledR}\right)$ /poligliceril 6-dioleato (Plurol Oleico $\left.^{\circledR}\right)$, miristato de isopropila e água para a proporção tensoativo:cotensoativo 2:1. A área delimitada representa a $M E G$

Após a incorporação do DDA, por simples solubilização, o sistema obtido apresentou-se com a mesma limpidez, transparência e isotropia, mostrando que o DDA não influenciou a tensão interfacial entre a fase aquosa e a oleosa, não perturbando a estabilidade termodinâmica do sistema.

\section{Caracterização físico-química da MEG}

A MEG foi caracterizada 48 h após sua fabricação e seus resultados encontram-se apresentados na Tabela 1. A eficiência de encapsulação do DDA foi de 101,16 $\pm 0,74 \%$, mostrando que a incorporação do fármaco foi completa. Foi visto também que a densidade relativa (DR) e o índice de refração não se alteraram com a incorporação do fármaco.

Tabela 1. Resultado da caracterização físico-química da MEG selecionada

\begin{tabular}{lcc}
\hline $\begin{array}{l}\text { Parâmetros } \\
\text { físico-químicos }\end{array}$ & $\begin{array}{c}\text { MEG } \\
\text { sem DDA }\end{array}$ & $\begin{array}{c}\text { MEG } \\
\text { com DDA }\end{array}$ \\
\hline $\begin{array}{l}\text { Teor de DDA } \\
\text { na MEG } \\
\left(\mu \mathrm{g} \mathrm{mL}^{-1}\right)\end{array}$ & - & $22,40 \pm 0,28$ \\
$\mathrm{pH}$ & $6,85 \pm 0,07$ & $7,11 \pm 0,01$ \\
$\begin{array}{l}\text { Condutividade } \\
\left(\mu \mathrm{cm}^{-1}\right)\end{array}$ & $24,73 \pm 1,6$ & $73,55 \pm 0,7$ \\
$\begin{array}{l}\text { Densidade relativa } \\
\left(\mathrm{g} \mathrm{L^{-1 }}\right)\end{array}$ & $1,0275 \pm 0,003$ & $1,029 \pm 0,01$ \\
$\begin{array}{l}\text { Índice de refração } \\
\text { Potencial zeta } \\
(\mathrm{mV})\end{array}$ & $1,413 \pm 0,003$ & $1,413 \pm 0,004$ \\
$\begin{array}{l}\text { Tamanho de } \\
\text { gotículas (nm) }\end{array}$ & $-21,083 \pm 1,37$ & $-25,48 \pm 1,46$ \\
\begin{tabular}{l} 
IPD* \\
\hline
\end{tabular} & $94,6 \pm 2,9$ & $123,4 \pm 6,7$ \\
\hline
\end{tabular}

* Índice de polidispersividade

De forma geral, o pH ideal de uma formulação é padronizado de acordo com o $\mathrm{pH}$ de estabilidade dos componentes ativos utilizados e o de tolerância biológica para produtos cutâneos $(5,5$ a $8,0) .{ }^{26} \mathrm{~A}$ formulação desenvolvida nesta pesquisa apresentou $\mathrm{pH}$ completamente compatível com o da pele $(6,85 \pm 0,07)$ e sem alterações significativas após adição do fármaco $(7,1 \pm 0,01)$ conforme demonstrado na Tabela 1.

Por outro lado, observa-se, a partir dos dados da Tabela 1, que a condutividade $(\sigma)$ foi modificada com a adição do DDA, visto que os valores na MEG sem o DDA foram inferiores aos valores da MEG com DDA. Este resultado era esperado, já que ocorreu a adição de um sal ao sistema.

Na Tabela 1 estão apresentados, também, os valores de $\zeta \mathrm{mV}$ das MEG sem DDA e MEG com DDA, na qual se podem observar valores negativos. Esses resultados estão em concordância com a literatura, ${ }^{27,28}$ os quais relacionaram análise de $\zeta \mathrm{mV}$ e $\mathrm{pH}$ de sistemas dispersos, observando-se que o $\zeta \mathrm{mV}$ tende a se tornar mais negativo com o aumento do $\mathrm{pH}$. Essa negatividade pode ser atribuída à possível presença de grupos carboxilatos na superfície das gotículas dispersas.

As análises do diâmetro das gotículas da fase interna da MEG (Tabela 1) demonstraram uma distribuição do tipo monomodal e homogênea das gotículas (Figura 2), a qual foi evidenciada pelo baixo IPD. A literatura ${ }^{18,29-32}$ considera que um sistema disperso para ser considerado ME é necessário que o tamanho de suas gotículas da fase interna esteja entre 10 e $150 \mathrm{~nm}$. Por isso, podemos estimar que o sistema estudado seja uma MEG, bem como podemos utilizá-la para realização dos estudos posteriores.

A adição do DDA causou um aumento no tamanho das gotículas, porém elas mantiveram sua isotropia e homogeneidade com um pe- 


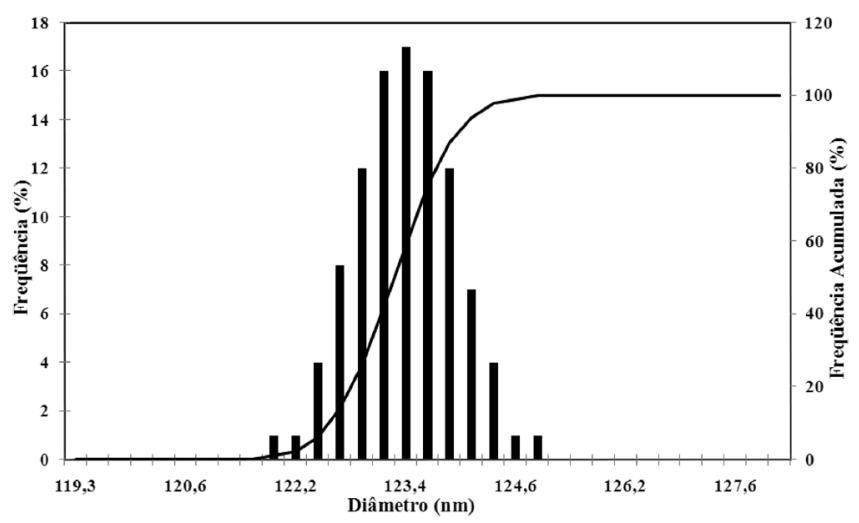

Figura 2. Distribuição do tamanho de gotículas por espalhamento de luz da MEG de DDA

queno IPD. O diâmetro das gotículas foi menor do que $200 \mathrm{~nm}$, sendo viável sua aplicação por via tópica ${ }^{33}$ já que a literatura descreve que sistemas que apresentam gotículas pequenas após a sua aplicação tópica podem ser utilizados como sistemas de liberação prolongada. ${ }^{34}$

\section{Estudo de liberação e permeação in vitro do DDA}

Na avaliação de produtos de ação dermatológica como, por exemplo, uma MEG de DDA, testes clínicos devem ser precedidos, e algumas vezes substituídos, por testes in vitro. Estes testes, devido às suas peculiaridades, permitem entender alguns fenômenos que ocorrem entre a aplicação do produto e o efeito medido farmacologicamente, de maneira prática, rápida e sem a interferência de fatores biológicos..$^{35}$

Para este estudo foi utilizada a célula de difusão baseada no modelo de Franz, usualmente empregada em estudo de liberação e permeação in vitro. É sabido que a eleição de uma solução receptora para os experimentos in vitro depende da solubilidade do fármaco, pois é necessária a manutenção das condições sink para garantir a obtenção de resultados efetivos. ${ }^{36}$ No caso do DDA, o mesmo mostrou-se muito solúvel em tampão fosfato $\mathrm{pH} 7,4$, com solubilidade superior à $6,0 \mathrm{mg} \mathrm{mL}^{-1} \mathrm{e}$, por isso, foi escolhido.

As Figuras 3 e 4 mostram os perfis de liberação e permeação in vitro da MEG e da formulação comercial emulgel genérico, usando membranas artificiais hidrofílicas de acetato de celulose (perfil cinético de $12 \mathrm{~h}$ ) e membrana da pele da orelha de porco (perfil cinético de $24 \mathrm{~h})$.

A taxa de liberação do DDA depende significativamente da afinidade do fármaco/veículo e da fração aquosa do sistema. ${ }^{15}$ Provavelmente, a liberação do DDA da MEG, comparada com o emulgel genérico, foi dificultada pelas combinações moleculares do DDA com a fração aquosa e interface do sistema, o qual causou um incremento da viscosidade formando uma malha gelificada que dificultou sua liberação. Esse resultado está em concordância com Djordjevic et al., ${ }^{15}$ que relatam que ME contendo um glicerídeo cáprico caprílico e poligliceril-6 dioleato, associado com miristato de isopropila, pode dificultar a liberação de drogas anfifílicas por interações moleculares.

Para calcular os parâmetros de liberação e permeação, em sistemas isotrópicos que apresentam propriedades difusionais em todas as direções como as ME, foi utilizada a primeira lei de Fick, obedecendo ao modelo de dose infinita, cujo gradiente de concentração é constante em consequência da concentração de fármaco do compartimento doador ser constante. ${ }^{26}$ Portanto, plotou-se quantidade liberada e permeada por área versus tempo, cuja representação gráfica está descrita nas Figuras 3 e 4.

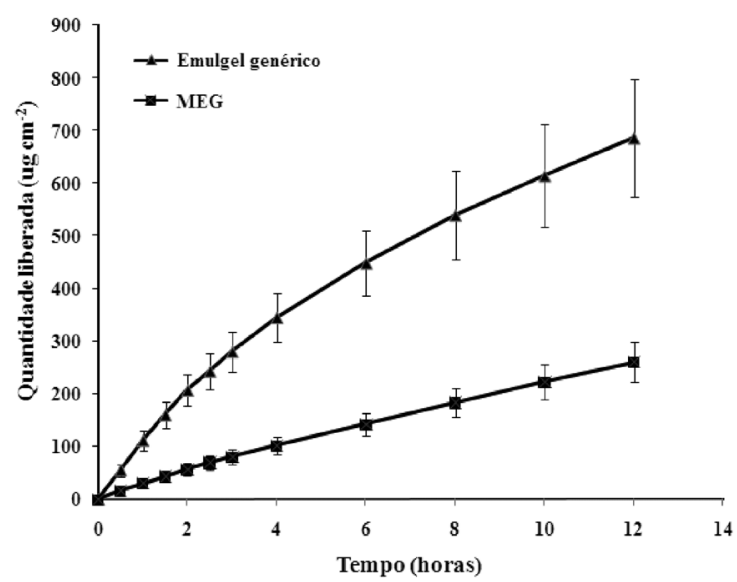

Figura 3. Quantidade liberada do diclofenaco de dietilamônio em membrana sintética de acetato de celulose da MEG e do emulgel comercial genérico

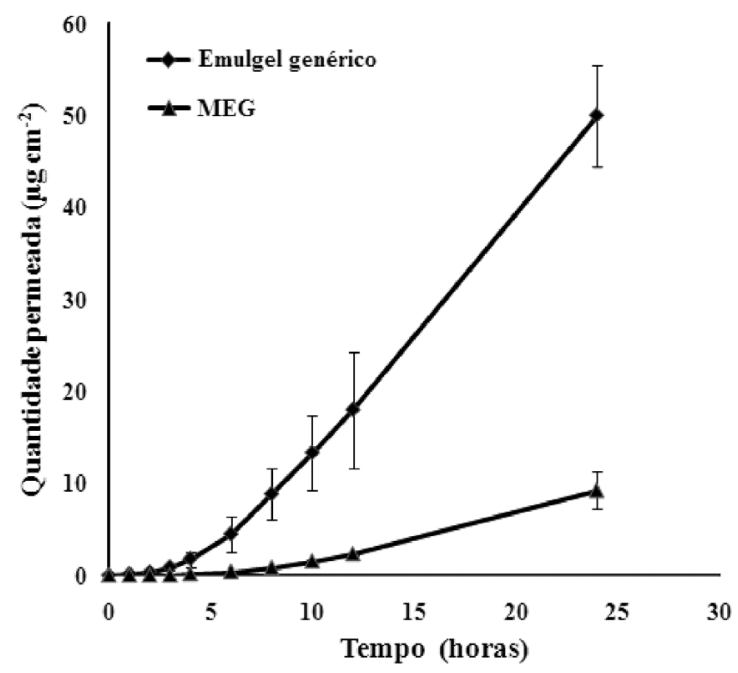

Figura 4. Quantidade permeada do diclofenaco de dietilamônio em pele da orelha de porco da MEG e do emulgel comercial genérico

Observa-se que houve uma diferença significativa entre as formulações testadas desde as primeiras horas dos perfis cinéticos, tanto em membrana artificial quanto em pele da orelha de porco. O $K_{p}$ da MEG estudada em membrana artificial foi $5,76 \times 10^{-3} \pm 7,1 \times 10^{-4} \mathrm{~cm} \mathrm{~h}^{-1}$, enquanto que o do emulgel genérico foi $1,28 \times 10^{-2} \pm 2,3 \times 10^{-3} \mathrm{~cm}$ $\mathrm{h}^{-1}$ (2,2 vezes maior). O mesmo comportamento foi observado para o $K_{p}$ usando membrana da orelha de porco, onde o emulgel comercial

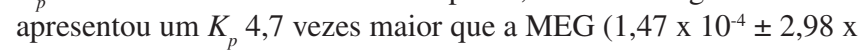
$10^{-5} \mathrm{~cm} \mathrm{~h}^{-1}$ para a MEG e $6,88 \times 10^{-4} \pm 6,91 \times 10^{-5} \mathrm{~cm} \mathrm{~h}^{-1}$ para o emulgel comercial). Esse menor $K_{p}$ da MEG estudada pode ser atribuído ao fato de que no emulgel comercial é utilizado um álcool em sua formulação, na qual atua como promotor de permeação de AINEs, fato que está em concordância com Bommannan et al.. ${ }^{37}$

Outro fator que deve ser levado em consideração para explicar o menor desempenho no perfil cinético da MEG está relacionado aos fenômenos de interface, ou seja, a atividade de superfície e micelização do DDA afetaram a sua liberação. Possivelmente, o DDA formou micelas o que ocasionou sua retenção na formulação, justificando o menor desempenho da MEG estudada.

As velocidades de liberação e, consequentemente, de permeação variam de acordo com as características das formulações, sendo que foi observado um aumento no tempo necessário (0-5 h) para que o $J$ das formulações emulgel genérico e MEG 
se equilibrassem, ou seja, ocorreu um tempo de latência (Figura 4). Decorrido o tempo de latência, foi possível definir o tipo de cinética de permeação cutânea como de ordem zero, ${ }^{35}$ pois as mesmas apresentaram um coeficiente de correlação ( $\mathrm{r}>0,98)$, indicando que o $J$ independe da concentração do fármaco. O tempo de latência pode ser definido como o tempo necessário para que o sistema entre em equilíbrio, o qual é calculado por extrapolação da reta de regressão linear até o eixo do tempo. ${ }^{38}$ A literatura ${ }^{25}$ relata que a inclinação da porção linear é considerada como correspondente ao estado estacionário de difusão, a qual representa o fluxo de penetração do fármaco através da pele $\left(\mu \mathrm{g} \mathrm{cm}^{-2} \mathrm{~h}^{-1}\right)$ e a sua interseção com o eixo da abscissa permite extrapolar o valor do tempo de latência, o que representa o tempo necessário para que a difusão do fármaco através da pele ocorra no chamado estado estacionário. Este resultado é muito relevante tendo em vista que o desejável é uma ação local e o perfil cinético encontrado, neste estudo, demonstra um incremento desta ação local.

A literatura relata que as ME têm grande capacidade de liberação de fármacos a partir da pele. ${ }^{1,30}$ No entanto, neste estudo, foi encontrado resultado contraditório, pois a MEG apresentou uma menor quantidade liberada e permeada, respectivamente, que o emulgel genérico (Figuras 3 e 4), fato que pode ser atribuído a uma diminuição da mobilidade do DDA incorporado, o que se traduziu numa difusão mais lenta e $J$ diminuído.

\section{CONCLUSÃO}

Apesar das MEs serem consideradas veículos capazes de promover a permeação de compostos na pele, resultados contrários foram obtidos neste estudo, onde a MEG estudada apresentou capacidade de permeação inferior a uma formulação convencional do mercado. Isto pode ser explicado através de uma maior retenção do fármaco na formulação, devido a uma interação molecular entre o DDA e os componentes da MEG. Entretanto, um estudo da modificação desta organização molecular é necessário para confirmar este mecanismo de retenção e poderá ser feito em estudo posterior. Desta forma, pode-se concluir que as ME contendo miristrato de isopropila, glicerídeos cáprico caprílico e poligliceril-6-dioleato não resultam em sistemas promotores de permeação tão efetivos na pele quanto a formulação comercial presente no mercado para o fármaco DDA. Por outro lado, este sistema demonstra uma grande capacidade em potencializar o efeito tópico da preparação, o que é um aspecto positivo em se tratando de um anti-inflamatório de uso local. Tal propriedade reduzirá consideravelmente os eventuais efeitos colaterais induzidos por uma distribuição sistêmica do DDA, quando comparado ao que o sistema comercial venha a produzir.

\section{AGRADECIMENTOS} UEPB.

\section{REFERÊNCIAS}

1. Ozguney, I. S.; Karasulu, H. Y.; Kantarci, G.; Sozer, S.; Guneri, T.; Eertan, G.; AAPS PharmSci. 2006, 7, E1.

2. Silva, J. A.; Tese de Doutorado, Universidade Federal de Pernambuco, Brasil, 2008.
3. Martins, M. R. F. M.; Veiga, F.; Rev. Bras. Ciênc. Farm. 2002, $38,33$.

4. Ke, W. T.; Lin, S. Y.; Ho, H. O.; Sheu, M. T.; J. Control. Release 2005, 102, 489.

5. Oliveira, A. G.; Scarpa, M. V.; Correa, M. A.; Cera, L. F. R.; Formariz, T. P.; Quim. Nova 2004, 27, 131.

6. Djordjevic, L.; Primorac, M.; Stupar, M.; Krajisnik, D.; Int. J. Pharm. 2004, 271, 11 .

7. Escribano, E.; Calpena, A. C.; Queralt, J.; Obach, R.; Domenech, J.; Eur. J. Pharm. Sci. 2003, 19, 203.

8. Mendonça, C. R. B.; Bica, C. I. D.; Piatnicki, C. M. S.; J. Braz. Chem. Soc. 2003, 14, 628.

9. Paolino, D.; Ventura, C. A.; Nistico, S.; Puglisi, G.; Fresta, M.; Int. J. Pharm. 2002, 244, 21.

10. Taha, M. O.; Al-Ghazawi, M.; Abu-Amara, H.; Khalil, E.; Eur. J. Pharm. Sci. 2002, 15, 461.

11. Sha, D. O.; Micelles, microemulsions and monolayers, Marcel Dekker, Inc: New York, 1998.

12. Jurkovic, P.; Gasperlin, M.; Farmacevtski Vestnir 2004, 55, 565.

13. Kriwet, K.; Mullergoymann, C. C.; Eur. J. Pharm. Biopharm. 1993, 39, 234.

14. Nokhodchi, A.; Sharabiani, K.; Rashidi, M. R.; Grafourian, T.; Int. J. Pharm. 2007, 335, 97.

15. Djordjevic, L.; Primorac, M.; Stupar, M.; Int. J. Pharm. 2005, 296, 73.

16. Formariz, T. P.; Chiavacci, L. A.; Sarmento, V. H. V.; Santilli, C. V.; Egito, E. S. T.; Oliveira, A. G.; Colloids Surf., B 2007, 60, 28.

17. Formariz, T. P.; Sarmento, V. H. V.; Silva-Junior, A. A.; Scarpa, M. V.; Santilli, C. V.; Oliveira, A. G.; Colloids Surf., B 2006, 51, 54.

18. Sintov, A. C.; Shapiro, L.; J. Control. Release 2004, 95, 173.

19. Moraes, S. L.; Rezende, M. O. O.; Quim. Nova 2004, 27, 701.

20. Silva, J. A.; Bedor, D. C. G.; Damasceno, B. P. G. L.; Oliveira, A. G.; Egito, E. S. T.; Santana, D. P.; J. Dispersion Sci. Technol. 2009, 30, no prelo.

21. Farmacopéia Brasileira, $4^{a}$ ed., Andrei editora: São Paulo, 2003.

22. Veríssimo, L. M.; Dissertação de Mestrado, Universidade Federal do Rio Grande do Norte, Brasil, 2007.

23. Lira, A. A. M.; Tese de Doutorado, Universidade de São Paulo, Brasil, 2007.

24. Panigrahi, L.; Pattnaik, S.; Ghosal, S. K.; AAPS PharmSci. 2005, 6, E167.

25. Cornelio, R.; Mayorga, P.; Lat. Am. J. Pharm. 2007, 26, 883.

26. Auton, M. E.; Delineamento de Formas Farmacêuticas, $2^{\mathrm{a}}$ ed., Artmed: Porto Alegre, 2005.

27. Washington, C.; Adv. Drug Deliv. Rev. 1996, 20, 131.

28. Wiacek, A. E.; Chibowski, E.; Colloids Surf., B 2002, 25, 55.

29. Lawrence, M. J.; Rees, D. G.; Adv. Drug Deliv. Rev. 2000, 45, 89.

30. Kreilgaard, M.; Adv. Drug Deliv. Rev. 2002, 54, S77.

31. Tenjarla, S.; Crit. Rev. Ther. Drug Carrier Syst. 1999, 16, 461.

32. Friberg, S. E.; J. Soc. Cosmet. Chem. 1990, 41, 155.

33. Lundberg, B. B.; Mortimer, B. C.; Int. J. Pharm. 1996, 134, 119.

34. Trota, M.; J. Control. Release 1999, 60, 399.

35. Sato, M. E. O.; Gomara, F.; Pontarolo, R.; Andreazza, I. F.; Zaroni, M.; Rev. Bras. Cienc. Farm. 2007, 43, 195.

36. Shah, V. P.; Maibach, H. I.; Topical drug biovailability, bioequivalence, and penetration, Plenum Press: New York, 2003.

37. Bommannan, D.; Potts, R. O.; Guy, R. H.; J. Control. Release 1991, 16, 299.

38. Bemvindo, C. S.; Dissertação de Mestrado, Universidade Federal do Rio de Janeiro, Brasil, 2006. 